\title{
DEFINING SEMANTIC LEVELS OF DETAIL FOR INDOOR MAPS
}

\author{
Jorge Chen \\ Department of Geography, University of California, Santa Barbara, USA - jorgechen@ geog.ucsb.edu
}

KEY WORDS: Indoor mapping, level of detail, city modeling, CityGML, GIS, BIM

\begin{abstract}
:
One major challenge in creating indoor maps involves defining their levels of detail or LODs. While a consensus has emerged that indoor maps have at least two types of LODs, semantic and geometric, questions remain regarding their nature, their partitioning, and their relationships with each other as well as with other forms of LOD. Since semantics deals with the meanings of things, semantic LODs (SLODs) deal with the definition, classification, and partitioning of mapped entities. Unlike geometric LODs that are amenable to automation, SLODs have a more qualitative nature that defies automation and requires the careful application of human judgment. This paper proposes a framework for organizing semantic LODs by first classifying them based on the tangibility of mapped entities (i.e., intangible open spaces versus tangible physical features comprised of building structures and equipment and furnishings) and then partitioning each class based on the idea of permanence, defined here as an entity's tendency to remain stationary over time. A cartographic process for integrating SLODs with geometric and appearance LODs is also introduced along with several examples.
\end{abstract}

\section{INTRODUCTION}

Cartographic level of detail, or LOD, describes the amount and type of graphical information that a map reveals at various views, scales, or "zoom" levels. While digitization has automated many aspects of the map production process, defining LODs remains one of the few areas that still elude automation and require the use of human judgment (Bertin, 2010). While most scholarly research on LODs has historically focused on two-dimensional (2D) and $2.5 \mathrm{D}^{1}$ maps of outdoor environments, research on characterizing LODs for indoor maps has attracted relatively little attention, perhaps due to the relative dearth of use cases, high quality indoor maps, and indoor mapping data.

In recent years, the success of ubiquitous outdoor maps has led to growing interest in the use of more sophisticated indoor maps in a wide range of applications, such as those listed in Table 1. This demand has led to technological and methodological advancements that are making indoor maps increasingly accessible — such as through BIM-GIS ${ }^{2}$ integration, precise indoor positioning, 3D indoor reality capture, web mapping, and integration into autonomous mobility devices-but the subject of indoor LODs remains largely unaddressed beyond a handful of studies for improving indoor LOD support in the City Geography Markup Language (CityGML).

This study builds on those CityGML-related works but expands the scope beyond CityGML to examine fundamental LOD principles for use in a wider range of indoor cartographic applications. Its focus is on the most subjective and qualitative aspect of LOD, namely semantics, which determines the inclusion or omission of mapped features based on their meanings (e.g., walls, furnishings, floor subdivisions, etc.). Since semantics makes up just one

\footnotetext{
${ }^{1} 2 \mathrm{D}$ plus height

${ }^{2} \mathrm{BIM}$ - building information modeling; GIS—geographic information systems

${ }^{3}$ Based on Dominguez et al. (2011); Open Geospatial Consortium (2012); Kemec et al. (2012); Zlatanova et al. (2013); Löwner et al. (2013); Kang et al. (2013); Kang and Lee (2014); Solou and Dimopoulou (2016); Tah et al. (2017); and Zlatanova and Isikdag (2017)
}

\begin{tabular}{ll}
\hline architecture & space planning \\
building design & $\begin{array}{l}\text { administration \& taxation } \\
\text { noise studies } \\
\text { facilities management }\end{array}$ \\
facility sensing \& monitoring & $\begin{array}{l}\text { energy planning } \\
\text { foot traffic analysis }\end{array}$ \\
resource management & indoor navigation \& guidance \\
indoor cadasters & emergency response \\
mining of urban metals & disaster simulation \& training \\
& gamification \\
\hline
\end{tabular}

Table 1. Potential uses for indoor maps ${ }^{3}$

aspect of LOD, this paper also proposes an approach for integrating it with geometric and appearance LODs. Therefore, the goals of this paper are, first, to present principles for establishing semantic LODs and then to propose a comprehensive framework for integrating them with the other two forms of LOD. For brevity, the acronyms SLOD, GLOD, and ALOD will be used for semantic, geometric, and appearance LODs, as shown in Table 2.

\begin{tabular}{lll}
\hline & Type of level-of-detail & Feature's property \\
\hline SLOD & Semantic level-of-detail & Meaning \\
GLOD & Geometric level-of-detail & Shape and geometry \\
ALOD & Appearance level-of-detail & Graphical appearance \\
\hline
\end{tabular}

Table 2. Level-of-detail acronyms used in this paper

\section{RELATED WORK}

Graphical visualization of indoor space covers a wide range of domains that span from architectural design to computer gaming. The domain that most closely aligns with cartography lies in the geographic information systems (GIS) and urban modeling domains, so the bulk of this review on LOD will focus on GIS-related works that center on CityGML. Since indoor mapping data can flow across domains, this review will also look at LOD concepts in building information modeling (BIM) as well as computer graphics, since the former can serve as a rich source of indoor mapping data and the latter for pure visualization. 


\subsection{CityGML}

Only a handful of studies have addressed indoor semantic LODs from a cartographic standpoint, most of which looked at shortcomings in the CityGML urban modeling standard. CityGML 2.0, the current version, provides five pre-defined levels of detail ranging from LOD0 (most generalized) to LOD4 (most detailed), as illustrated in Fig. 1. LOD4 is externally identical to LOD3 but adds indoor content, while LOD0 to LOD3 show empty space for a building's interior. This all-or-nothing approach to indoor features severely limits its usefulness for indoor mapping and has led to several proposals to further partition LOD4. However, as adaptations of an existing standard, all of these approaches were constrained by the five LOD framework of CityGML, which in most cases forced the coupling of semantics and geometry.

Exterior

LOD0

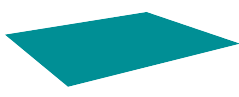

LOD1
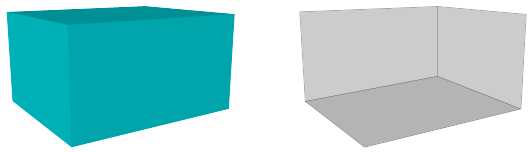

LOD2
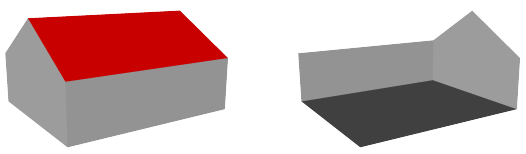

LOD3
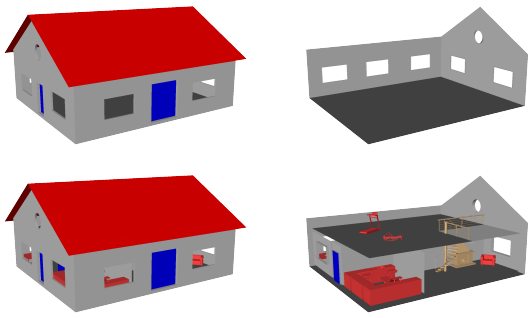

Figure 1. Example of LODs for CityGML. Model courtesy of Applied Computer Science at Karlsruhe Institute of Technology.

One group of approaches started with the semantic feature of the floor or story and progressively added both semantic and geometric details. Billen et al. (2012) used a volumetric representation of subspaces within a floor (e.g., rooms and hallways) and added details with interior openings followed by exterior ones before reaching LOD4. Hagedorn et al. (2009) started at a more generalized level of the entire floor using 2D geometry (i.e., the floor footprint) followed by 2D floor plans showing partitioned spaces, 3D models with a selection of indoor landmarks, and then LOD4. Similar to Hagedorn et al., Kemec et al. (2012) also started with the entire floor but used 3D geometry instead; they then added a level containing functional spaces before adding rooms and corridors and moving to LOD4.

Instead of using digital models, Kang and Lee (2014) and Jung et al. (2016) started with raster images of floor plans. They then added details using spherical omni-directional photographs followed by a simplified 3D indoor model before reaching LOD4. Jung et al. (2016) also added 2D computer-aided design (CAD) floor plans after the raster floor plans. The imagery-based portions of these two approaches resemble the Street View feature in Google Earth and Google Maps.

\begin{tabular}{|c|c|}
\hline Billen et al. (2008) & Hagedorn et al. (2009) \\
\hline $\begin{array}{l}\text { - Partitioned spaces (Polyhedra) } \\
\text { - Add interior openings } \\
\text { - Add exterior openings } \\
\text { - Full interior }\end{array}$ & $\begin{array}{l}\text { - Floors (2D floor footprint) } \\
\text { - Partitioned spaces (2D floor plans) } \\
\text { - 3D model with landmarks } \\
\text { - Full interior }\end{array}$ \\
\hline \multicolumn{2}{|l|}{ Kemec et al. (2012) } \\
\hline $\begin{array}{l}\text { - Floors (Polyhedra) } \\
\text { - Functional spaces (Polyhedra) } \\
\text { - Partitioned spaces (Polyhedra) } \\
\text { - Full interior }\end{array}$ & \\
\hline Kang and Lee (2014) & Jung et al. (2016) \\
\hline $\begin{array}{l}\text { - Floor plan image } \\
\text { - Omni-directional image } \\
\text { - Simplified 3D model } \\
\text { - Precise 3D model }\end{array}$ & $\begin{array}{l}\text { - Floor plan image } \\
\text { - 2D CAD } \\
\text { - Panoramic image } \\
\text { - Simple geometry \& texture } \\
\text { - Full interior }\end{array}$ \\
\hline
\end{tabular}

Table 3. Summary of prior proposals for indoor versions of CityGML. Indoor LODs are listed from lowest to highest.

The preceding proposals used a strict coupling between semantics and geometry; that is, for a given LOD, a semantic feature such as a door could only be represented by a single geometric form, such as a $3 \mathrm{D}$ polyhedron or $2 \mathrm{D}$ line drawing. Benner et al. (2013) and Löwner et al. (2013) proposed an alternative approach that decoupled semantics from geometry, which made it possible to produce multiple combinations of semantic and geometric LODs. Working within the constraints of CityGML, they proposed semantic LODs that progressed from boundary surfaces to surface openings (e.g., doors and windows) and geometric LODs that progressed from 2D simplifications to exact 3D models, as summarized in Fig. 2 and Table 4, noting that Löwner et al. also added furniture to their SLOD framework. Even though these four SLODs and four GLODs can provide 16 different combinations, they restricted them to ten as shown in Fig. 3 citing incompatible combinations, such as vertical extrusions (GLOD1) of door or window openings (SLOD3).

\begin{tabular}{l|cccc|cccc}
\hline Indoor semantic & \multicolumn{3}{|c|}{ Benner et al. } & \multicolumn{4}{c}{ Löwner et al. } \\
levels of detail & 0 & 1 & 2 & 3 & 0 & 1 & 2 & 3 \\
\hline Boundary surfaces & & $\bullet$ & & & & $\bullet$ \\
Building installations & & & $\bullet$ & & & & $\bullet$ \\
Furniture & & & $\bullet$ & & & & & \\
Surface openings & & & & $\bullet$ & & & \\
\hline
\end{tabular}

Figure 2. Indoor semantic levels of detail as proposed by Benner et al. (2013) and Löwner et al. (2013)

\begin{tabular}{ll}
\hline GLOD0 & 2D or 2.5D geometry \\
GLOD1 & Solid vertical extrusion \\
GLOD2 & Generalized 3D geometry \\
GLOD3 & Exact 3D geometry \\
\hline
\end{tabular}

Table 4. Geometric levels of detail as proposed by Benner et al. (2013) and Löwner et al. (2013)

\subsection{BIM}

openBIM, the de facto baseline standard for building information modeling (BIM), uses an LOD approach that is not amenable to cartography but is mentioned here since BIM models can serve as a rich source for indoor mapping data. BIM has recently emerged as the prevailing $3 \mathrm{D}$ modeling process for the architecture, engineering, and construction (AEC) industry (Succar, 2009), and its 


\begin{tabular}{lcccc}
\hline & SLOD0 & SLOD1 & SLOD2 & SLOD3 \\
\hline GLOD0 & $\bullet$ & $\otimes$ & $\otimes$ & $\otimes$ \\
GLOD1 & $\bullet$ & $\otimes$ & $\otimes$ & $\otimes$ \\
GLOD2 & $\bullet$ & $\bullet$ & $\bullet$ & $\bullet$ \\
GLOD3 & $\bullet$ & $\bullet$ & $\bullet$ & $\bullet$ \\
\hline
\end{tabular}

--allowed $\otimes$-prohibited

Figure 3. Permissible combinations of SLOD and GLOD per Benner et al. (2013) and Löwner et al. (2013)

concept of LOD—called level of development and designated as $L O D t$ in this paper-reflects the various stages of facility design and construction. The American Institute of Architects (AIA) publishes the LODt standard, which has six LODts (100, 200, $300,350,400$, and 500) corresponding to the progressive levels of confidence in the actual state of a building's construction (Bedrick et al., 2014; Reinhardt and Bedrick, 2016). LODt 100 corresponds to the lowest level of confidence often encountered at project inception while LODt 500 should show the actual state of construction, often referred to as the as-built or as-is state, as illustrated in Fig. 4.

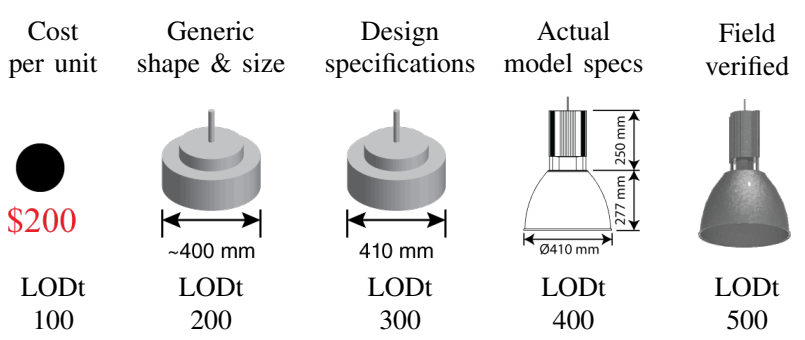

Figure 4. Example of LODts for a light fixture. Models for LODts 400 and 500 courtesy of the Luminaires Group.

\subsection{Computer graphics}

Computer graphics (CG) formats use a geometric LOD approach based on polygon counts to optimize efficient rendering for visualization, as illustrated in Fig. 5 (Luebke et al., 2003). Some CG formats offer no semantic capabilities while others offer a primitive form of semantics to represent grouped geometric shapes, both of which fall short of semantically relating geometry to realworld features.

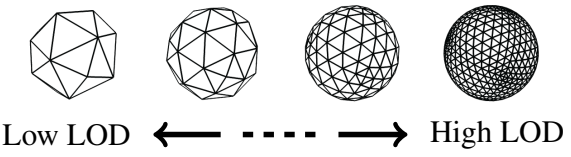

Figure 5. Levels of detail of a sphere in computer graphics

\subsection{Assessment}

CityGML's five-LOD framework was developed to standardize the modeling of city environments for monetizing urban spatial information (Brüggemann and Liebig, 2000), but this framework deals mostly with the outdoors and significantly limits indoor mapping capabilities, even with LOD4 and its proposed revisions. First, the standard offers only a single indoor LOD and attempts to subdivide LOD4 are limited by the five LOD framework, which oftentimes imposes strict semantic-geometric coupling. Second, CityGML obscures the distinction between tangible physical features and intangible occupied space when it moves from footprint and extruded volume (LOD0 and LOD1) to the physical form of the building (LOD2, LOD3, and LOD4). This arbitrary break prevents further semantic decomposition of occupied space to finer granularities (e.g., that occupied by furnishings) and further geometric generalization of physical features to coarser granularities. Finally, CityGML's single indoor LOD constrains geometric representations to resemble the actual form of semantic objects; for example, a door must always assume a rectangular form but may not use a more abstract representation, such as a sphere.

openBIM's use of LODt and its limited semantic LOD capabilities reflect its intended use in facility design and construction. Moving from LODt 500 to 100 does not always result in progressive geometric generalization. For instance, moving from LODt 300 to 500 may result in the same amount of geometric detail but only reflect changes in dimensions as a project moves from design to actual construction, and going from LODt 400 to 500 may even result in a loss of detail under some implementations. Regarding semantic LODs, openBIM provides formally published Model View Definitions (MVDs) that extract subsets of features from the standard Industry Foundation Classes (IFC) data model. However, they cannot be modified and are intended more for software development than map design. In short, openBIM does not support geometric generalizations nor does it provide flexibility with semantic LODs. These observations apply to most other BIM implementations, which use openBIM as a template.

In summary, neither CityGML nor BIM offers the capability for richly supporting semantic LODs, while most CG formats offer none. For visualization, CityGML and most CG formats support geometric generalization insofar as the generalized form of a feature resembles its exact form, and openBIM offers only a single geometric LOD per LODt; none allows use of alternative geometries. Notably, CityGML and BIM assign semantics based on a feature's actual shape, e.g., something shaped like a door is called a door, but they provide no further abstractions. Finally, from a practical standpoint, high costs associated with gathering high fidelity indoor data means that very few LOD4 data sets actually exist.

\section{INDOOR SEMANTIC LEVELS OF DETAIL}

\subsection{Core concepts}

Before laying out the proposed framework for indoor semantic LOD, it may be helpful to review and establish some core concepts and definitions. First, this paper uses the term semantics to refer broadly to the abstract "meaning of things" (Hu, 2018). For instance, a vertical partition can be semantically defined as a "wall" while the semantic term "food court" can define an abstract cluster of restaurants in a shopping mall.

Second, the abstract semantics of an object is defined here as being completely detached from its physical form, extending the work started by Becker et al. (2013), Löwner et al. (2013), and others to decouple semantics from geometry. Whereas the previous authors derived semantics from a feature's geometry but still maintained a link to its original physical form when changing the geometric LOD (i.e., original geometry $\Leftrightarrow$ semantics), this paper takes the process one step further by allowing a semantic entity to assume any geometric form or even none at all (i.e., original geometry $\Rightarrow$ semantics $\Rightarrow$ any geometry). Under this framework, the semantic "food court" entity from the previous example can 
have any number of geometric expression, e.g., 2D line drawings, $3 \mathrm{D}$ polyhedra, a raster image, or even a simple sphere.

Third, the separation of semantics from geometry results in a semantic LOD and a geometric LOD, while further refinement adds an appearance LOD, resulting in a total of three types of LODs (Löwner et al., 2013). Since geometry must have appearance properties to be visualized (e.g., color, transparency, etc.), the appearance LOD is attached to the geometric LOD to control these visualization parameters. Fig. 6 shows these relationships.

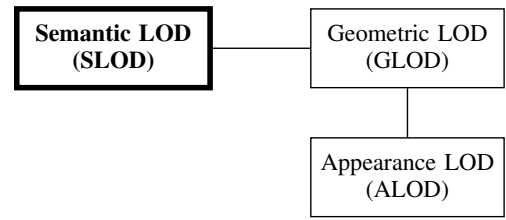

Figure 6. Integration of SLOD, GLOD, and ALOD

Finally, this paper introduces the concept of permanence for guiding the subdivision of semantic LODs. Here, permanence is defined as an entity's tendency to remain unchanged over time, which can be seen as a measure of temporal stability or temporal inertia. It has a simple concept: some indoor entities tend to remain more stationary over time than others, with the more stationary ones tending to occupy the background in a Gestalt figure-ground perception hierarchy. Using CityGML as an example, the footprint of a building (LOD0) will be least likely to change over time compared to the arrangement of room partitions and the locations of furnishings (LOD4). When visualized, room partitions and furnishings will have greater salience (i.e., they become the figure) while the building footprint will recede into the background.

\subsection{Three classes of semantic LODs}

How can a building's interior be partitioned based on semantics alone? As a starting point, a distinction can be made between physical objects and the 2D or 3D space they occupy (Kemec et al., 2012; Zlatanova et al., 2016), since one has tangible properties and the other does not. This leads to two semantic classes: physical features and occupied space. The physical features can be further subdivided into the essential and nonessential elements of a building, with the former classified as the structural elements that hold the building together and define its indoor environment and the latter as equipment and furnishings that adds functionality to the indoor space. This taxonomy results in three semantic LOD classes consisting of occupied space, building structure, and equipment and furnishings, as illustrated in Fig. 7. LOD levels are then defined using permanence for each of these SLOD classes.

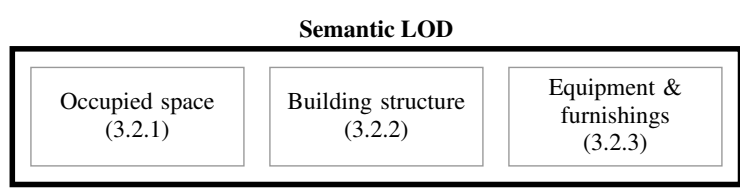

Figure 7. The three classes of semantic LOD

3.2.1 Occupied space SLODs Occupied space possesses an abstract and intangible form that can coincide with both the physical and imaginary boundaries of an indoor environment. For example, applying permanence to indoor physical boundaries can result in three increasingly generalized SLODs for occupied space: individual rooms and corridors, entire floors, and the entire building interior. Here, the semantics of rooms and corridors will change most often through activities such as the installation or removal of partition walls or changes in function (e.g., hair salon to restaurant). Likewise, the semantics of floors can be redefined, such as through the installation of mezzanines or by changing floor numbers (e.g., removing 4 or 13 in certain cultures), though these would happen less frequently than room and corridor changes. However, the overall interior of the building will likely remain unchanged regardless of how often floors, rooms, and corridors get redefined.

This three-SLOD division of occupied space only serves as an example. Other possibilities include the addition of functional spaces, space occupied by "clutter" for use in navigation, etc. For illustration purposes, however, this paper will continue to use the three subdivisions, designated as S1 (entire building interior), S2 (entire floors), and S3 (rooms and corridors), as shown in Fig. 8.

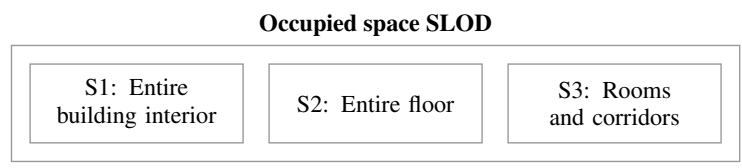

Figure 8. Levels of occupied space SLOD

3.2.2 Building structure SLODs The building structure contains almost everything conventionally considered real property ${ }^{4}$, including floors, ceilings, walls, and appurtenances (e.g., mouldings, doors, and windows), but it excludes real property installed equipment, which is moved to the equipment SLOD. Structural features with the greatest permanence generally consist of loadbearing members that have the least likelihood of being modified due to cost and complexity; these can include features such as concrete columns, floor slabs, and load-bearing walls. Non-load bearing structural members follow next, which mainly consist of partition walls that may be modified multiple times during the life of a facility. Finally, appurtenances such as doors and windows have the least permanence, since they are oftentimes replaced or modified on a more frequent basis.

This taxonomy results in three structural SLODs from the most permanent to the least: structural load-bearing members, nonload bearing members, and structural appurtenances. Whereas CityGML places surfaces and openings in different LODs, the two structural LODs in this framework include openings as part of a structure's definition (e.g., for walls, floors, and ceilings). Again, for illustration purposes, this paper designates the structure SLODs as B1 (structural load-bearing members), B2 (nonload bearing members), and B3 (structural appurtenances), as illustrated in Fig. 9.

\begin{tabular}{|c|c|c|}
\hline \multicolumn{3}{|c|}{ Building structure SLOD } \\
\hline $\begin{array}{l}\text { B1: Structural } \\
\text { load-bearing } \\
\text { members }\end{array}$ & $\begin{array}{l}\text { B2: Non-load } \\
\text { bearing members }\end{array}$ & $\begin{array}{l}\text { B3: Structural } \\
\text { appurtenances }\end{array}$ \\
\hline
\end{tabular}

Figure 9. Levels of building structure SLOD

3.2.3 Equipment and furnishings SLODs Equipment and furnishings includes all inanimate physical entities that do not belong to the building structure. This paper divides equipment into three sub-classes comprised of real property installed equipment (RPIE), non-real property installed equipment (non-RPIE),

\footnotetext{
4 "United States Code of Federal Regulations," Definition of real property, Title 26, Sec. 1.856-10
} 
and furnishings, based roughly on their permanence qualities. By convention, RPIE includes equipment that is permanently integrated with the building structure and that serves an essential function, e.g., providing electricity, water, or heating and cooling. Examples of RPIE include elevators, boiler plants, and permanent walk-in refrigerators. Non-RPIE consists of all other static equipment that can be readily removed and that serve non-essential functions, such as washing machines, kitchen cabinets, and windowmounted air conditioners. ${ }^{5}$ Finally, furnishings consist of all other objects that can be readily moved, such as tables, chairs, window curtains, and office stationary. For illustration, this paper designates these SLODs as E1 (RPIE), E2 (non-RPIE), and E3 (furnishings), in order of most permanent to least, as illustrated in Fig. 10.

\begin{tabular}{|c|c|c|}
\hline \multicolumn{3}{|c|}{ Equipment SLOD } \\
\hline $\begin{array}{l}\text { E1: Real property } \\
\text { installed equipment }\end{array}$ & $\begin{array}{l}\text { E2: Non-real } \\
\text { property installed } \\
\text { equipment }\end{array}$ & E3: Furnishings \\
\hline
\end{tabular}

Figure 10. Levels of equipment and furnishings SLOD

\subsection{SLOD summary}

This SLOD framework decomposes the semantics of an indoor space based on the form of the entities being modeled (i.e., intangible space versus physical features) and their levels of permanence, resulting in the semantic levels of detail shown in Table 5. Of the three SLOD classes, occupied space has the most abstract form and thus the greatest latitude for modification, e.g., adding levels for functional space and space occupied by furnishings. However, adding, deleting, or modifying SLOD classes should have no impact on the other forms of LOD due to decoupling.

\begin{tabular}{ll}
\hline SLOD & Feature type \\
\hline S & Occupied space \\
- S1 & Entire building interior \\
- S2 & Entire floors \\
- S3 & Individual rooms and corridors \\
B & Building structure \\
- B1 & Structural load-bearing members \\
- B2 & Non-load bearing members \\
- B3 & Structural appurtenances \\
& (e.g., mouldings, doors, windows, etc.) \\
E & Equipment \& furnishings \\
- E1 & Real property installed equipment (RPIE) \\
- E2 & Non-real property installed equipment (Non-RPIE) \\
- E3 & Furnishings \\
\hline
\end{tabular}

Table 5. Semantic LODs

\section{AN INTEGRATED LOD FRAMEWORK}

Combining SLODs with the other forms of LOD provides a significant amount of flexibility for communicating and expressing ideas through the graphical language of maps (Bertin, 2010; Slocum et al., 2010; Nyerges, 1991). The cartographic process starts with identifying the subject of the map, which are the semantic entities, followed by their expression in visual geometric form and their spatial arrangement in either geometric or topological space. This paper will only address SLOD integration with

\footnotetext{
${ }^{5}$ United States Air Force. 2015. "Air Force Instruction 32-9005: Real Property Accountability and Reporting."
}

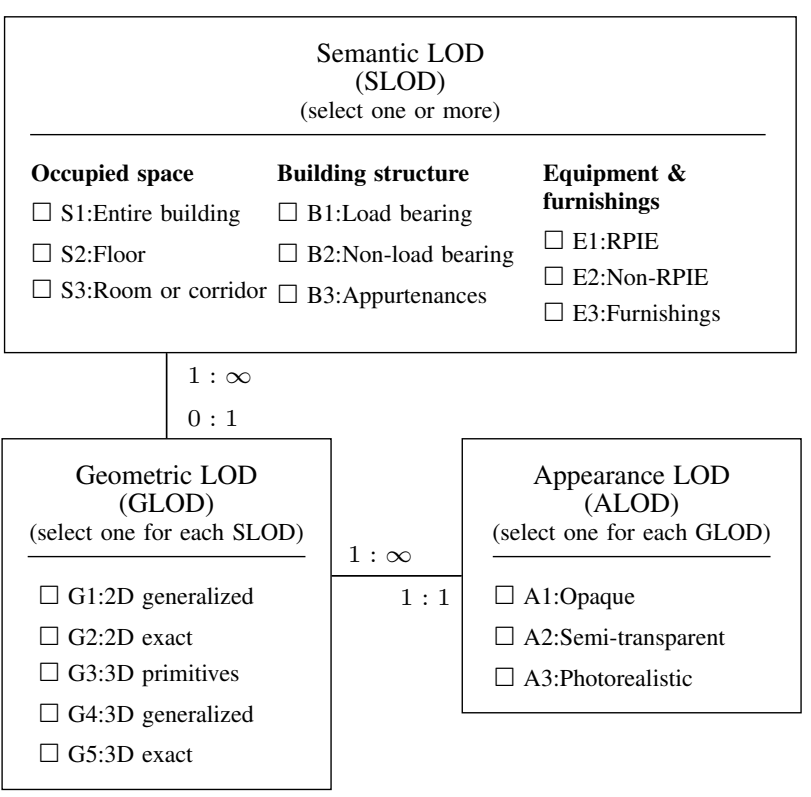

Figure 11. Integration of SLOD, GLOD, and ALOD

geometric LOD and, by extension, appearance LOD but will not cover topology.

\subsection{Geometric and appearance LODs}

Geometric and appearance LODs provide the graphical symbolism that gives maps their characteristic "visual narrative" qualities. In their decoupled approach to CityGML, Benner et al. (2013) and Löwner et al. (2013) proposed four geometric LODs: $2 \mathrm{D}$ or $2.5 \mathrm{D}$ geometry, solid vertical extrusions, generalized $3 \mathrm{D}$ geometry, and exact 3D geometry. However, they provided no proposal for an appearance LOD framework, although Löwner et al. acknowledged its relevance. ${ }^{6}$

This paper modifies their geometric LODs to include 2D generalized geometry, 2D exact geometry, 3D primitives, 3D generalized geometry, and 3D exact geometry, in order of increasing detail, as shown in Fig. 11. This new framework provides three improvements: first, the "3D generalized geometry" LOD incorporates the former 2.5D geometry and solid vertical extrusion LODs; second, a new "3D primitives" LOD has been added to provide a higher level of abstraction, e.g., representing a rectangular door as a sphere; and finally, removal of the $2.5 \mathrm{D}$ and vertical extrusion geometries eliminates any chance of incoherent semanticgeometric pairings. A simple three-level appearance LOD is also introduced consisting of monochromatic opaque color, monochromatic semi-transparent color, and photorealistic texturing; this adds the semi-transparent LOD to the other two already available in CityGML. This new framework, illustrated in Fig. 11, allows for any combination of semantics and geometry to exist without coherence problems.

\subsection{Non-literal representations}

Whereas technical renderings, e.g., CAD and BIM, attempt to capture the exact form and location of indoor features, maps of-

\footnotetext{
${ }^{6}$ It should be noted that CityGML uses the term appearance to describe the software implementation of texturing processes from the X3D and COLLADA specifications. What this paper calls appearance, CityGML calls portrayal, which it defers to "viewer applications or styling specification languages" to specify (Open Geospatial Consortium, 2012). This paper will continue to use the term appearance to describe the "look" of an object.
} 
tentimes distort reality or "lie" to effectively communicate their messages. As Monmonier (1996) noted, "Not only is it easy to lie with maps, it's essential. To portray meaningful relationships for a complex, three-dimensional world ... a map must distort reality." In this framework, distortions can occur through geometric generalization, alternative geometries, and changes in appearance. Notably, the use of alternative geometries is a feature not previously found in other proposals.

Another form of distortion involves concealing features by removing them or distorting surrounding features. For instance, visitors to a shopping mall will have no interest in the locations of janitor's closets; such information clutter can only detract from a map. Similarly, it may be desirable to completely remove sensitive locations from a public facing map, such as rooms containing servers, surveillance equipment, or high value items. Fig. 12 shows the concealment of a room from a public facing floor plan.

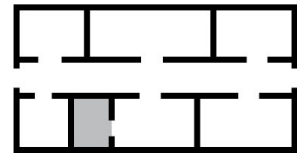

(a) Before

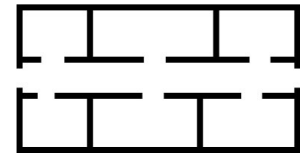

(b) After
Figure 12. The room shown in gray in floor plan (a) has been concealed in floor plan (b)

\subsection{Examples}

Occupied space Figure 13 shows an illustration of the various semantic levels of occupied space and how they can be rendered in either 2D or 3D geometric form. Though not shown, decoupling also allows the mixing of semantic and geometric forms, such as the $3 \mathrm{D}$ envelope of the entire building with 2D rooms and corridors. Mixing several types of SLODs is also possible as illustrated in the bottom left figure of Fig. 13, which shows the occupied space SLOD being used to highlight a physical model of a room (i.e., building structure and equipment and furnishings).

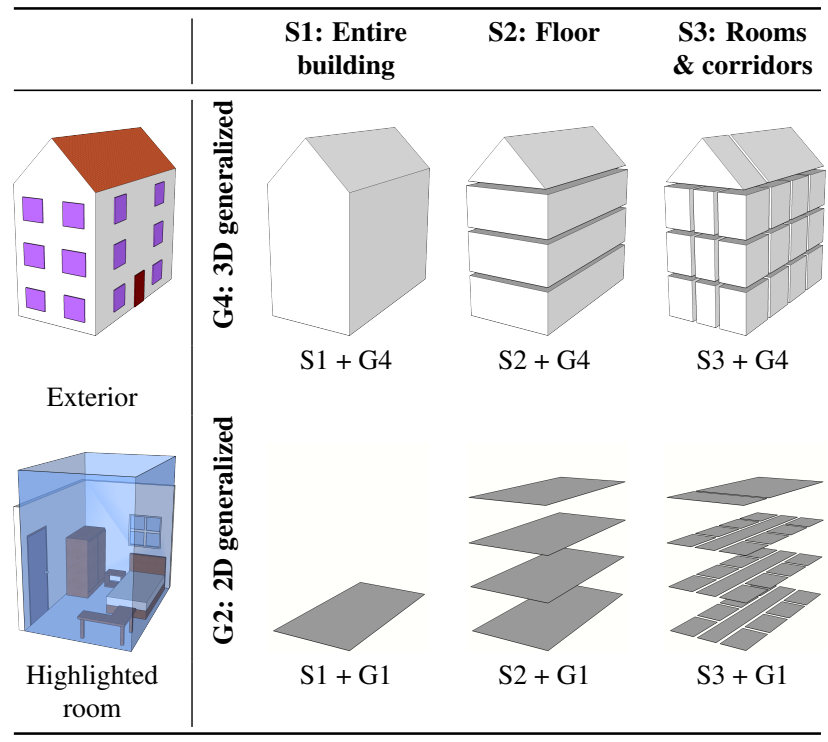

Figure 13. Occupied space with 2D and 3D geometries

Building structure and equipment and furnishings Figure 14 shows examples of different SLODs for a building structure, how they can be used both together and independently, and how changing the appearance LOD can be used to highlight features.
Similarly, Figure 15 shows examples for individual and combined levels of the equipment and furnishings SLOD class.

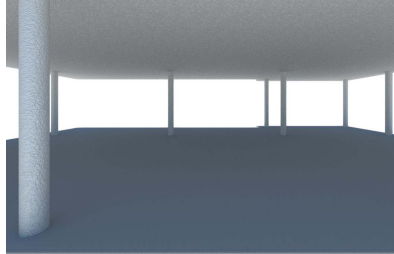

Load bearing structure (S1)

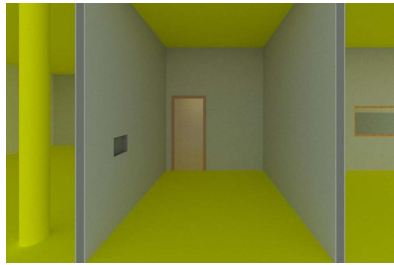

Load bearing structure in yellow

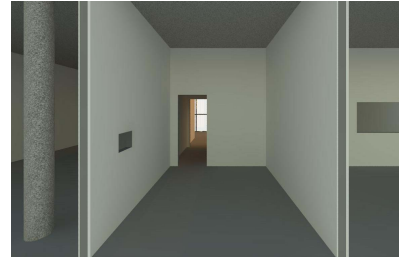

Non-load bearing structure (S2)

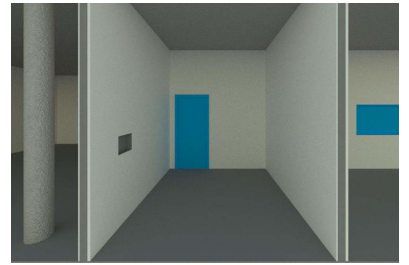

Structural appurtenances in blue
Figure 14. Indoor structure SLODs. Data source: Autodesk.

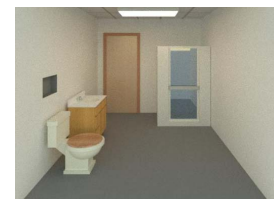

RPIE (E1)

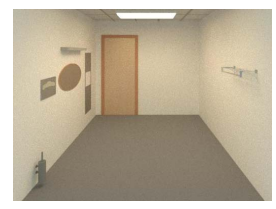

Non-RPIE (E2)

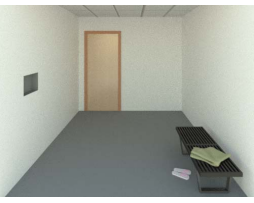

Furnishings (E3)

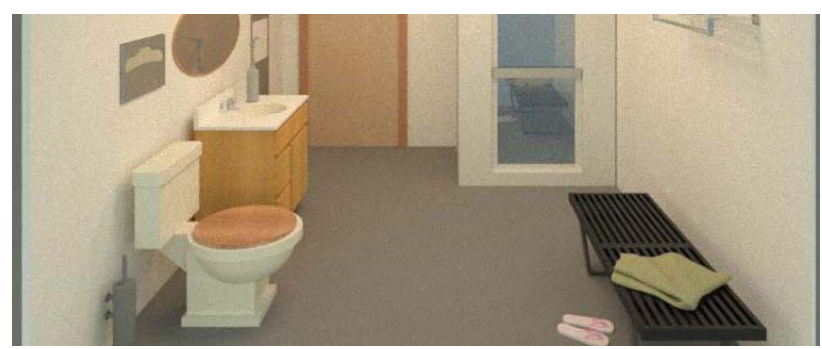

All equipment and furnishings SLODs combined (E1+E2+E3)

Figure 15. Indoor equipment and furnishings SLODs.

Data courtesy of Autodesk, RevitCity, Tork, and Kolpak.

Advanced cartographic expressions Figure 16 shows examples of how varying the semantic, geometric, and appearance LODs can produce advanced forms of cartographic expression. The simplified "see through walls" in Fig. 16a may be helpful for emergency response or law enforcement, while the highlighting of RPIE in Fig. 16b may be used by engineers, facility managers, and resource managers for facility planning.

\section{CONCLUSION}

Cartographic indoor mapping remains a relatively new and unexplored field of study. The semantic LOD framework presented in this paper builds on cartographic concepts from CityGML as well as several proposals for improving its indoor mapping capabilities, but it digresses in two important ways. First, whereas CityGML-based approaches placed geometry at the center of map design, this framework uses semantics as the map's foundational 


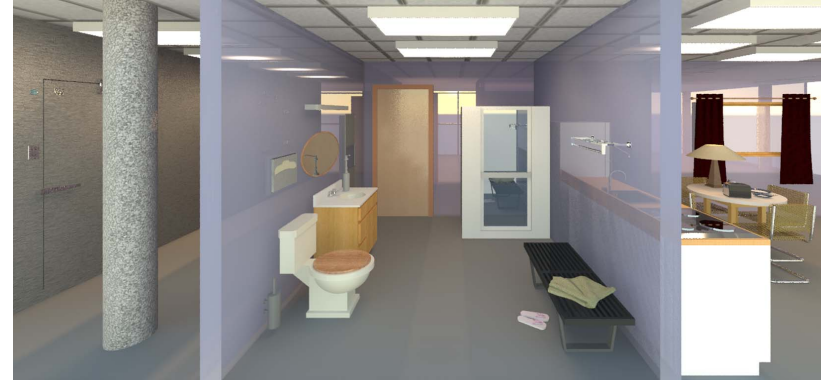

(a) Photorealistic rendering with abstract "see through walls"

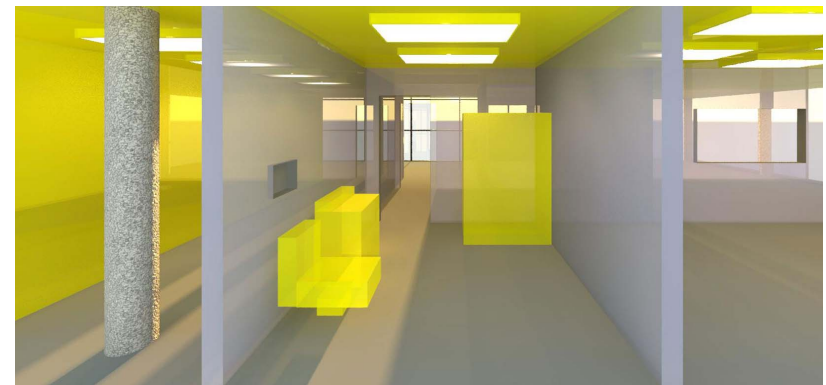

(b) Abstract rendering highlighting real property installed equipment

Figure 16. Advanced cartographic expressions using mixed LODs. Data courtesy of Autodesk, RevitCity, Tork, and Kolpak.

element and places geometry, topology, and other mappable properties in subordinate roles. Second, this framework completely removes the constraint of using fixed semantic-geometric LOD representations; instead, any combination of LODs can be used, including multiple instances from the same SLOD class. The advantages of using this more abstract and unconstrained approach includes greater flexibility and expressive freedom, but it comes at the cost of greater complexity. This framework is thus intended more for map designers and developers rather than casual endusers. Furthermore, this framework is not intended to replace CityGML, BIM, or any other established standard. Rather, it is intended to serve as a starting point for exploring basic principles of cartographic indoor mapping, with the long-term goal of finding better ways to create more sophisticated and effective maps of indoor spaces.

As an initial proposal, this SLOD framework has several potential shortcomings and opportunities for improvement. First, the SLODs can be more fully and rigorously developed beyond the three semantic classes presented in this study. This may involve assembling a more coherent taxonomy of occupied space to include other areas such functional spaces, structural spaces, and clutter. It may also involve adding classes for other building components, such as electrical and mechanical, while keeping in mind the need to preserve simplicity. Second, a semanticstopology relationship can be developed to support non-visual elements, such as for routing and navigation, that can have the added benefit of facilitating IndoorGML integration. This may include investigating how topological levels of detail (Hsu and Hsu, 2005) can apply to cartography. Finally, this framework can include SLODs for raster data (both 2D images and 3D voxels) and point clouds. 2D images provide an intuitive way to view the environment as observed by Kang and Lee (2014) and Jung et al. (2016), while indoor measurements often come in the form of point clouds, such as from laser scanners and structured light devices. Companies such as Indoor Reality ${ }^{7}$, Trimble $^{8}$, and Matterport ${ }^{9}$ have successfully integrated spherical images with point clouds in their commercial indoor mapping products, while the video game Minecraft has demonstrated the popular appeal of voxels (Zhu and Heun, 2017); an improved SLOD framework can help integrate these proven ideas in future indoor maps. Indoor mapping will continue to gain greater relevance as the demand for ubiquitous location-based services grows and as indoor reality capture techniques continue to improve. Having a flexible and coherent set of basic indoor mapping principles will help ensure the successful development of indoor maps in years to come.

\section{ACKNOWLEDGEMENTS}

This research was funded by the National Geospatial-Intelligence Agency's Academic Research Program (Award No. HM047617-1-2002, Project Title: Automated Level of Detail and Feature Extraction for 3D Indoor Mapping and Modeling). Approved for public release, 18-555. All unattributed graphics and images were produced by the author.

\section{References}

Becker, S., Peter, M., Fritsch, D., Philipp, D., Baier, P. and Dibak, C., 2013. Combined Grammar for the Modeling of Building Interiors. In: ISPRS Annals of Photogrammetry, Remote Sensing and Spatial Information Sciences, Vol. II-4/W1, pp. 1-6.

Bedrick, J., Jizba, A. and Vandezande, J., 2014. Exploring the LOD Specification. AIA KnowledgeNet Webinar.

Benner, J., Geiger, A., Gröger, G., Häfele, K.-H. and Löwner, M.O., 2013. Enhanced LOD concepts for virtual 3D city models. In: ISPRS Annals of Photogrammetry, Remote Sensing and Spatial Information Sciences, Vol. II-2/W1, pp. 51-61.

Bertin, J., 2010. Semiology of Graphics: Diagrams, Networks, Maps. 1st edn, ESRI Press : Distributed by Ingram Publisher Services, Redlands, Calif.

Billen, R., LaPlanche, F., Zlatanova, S. and Emgard, L., 2008. Vers la création d'un méta-modèle générique de l'information spatiale 3D urbaine. XYZ (114), pp. 37-42.

Billen, R., Zaki, C.-E., Servières, M., Moreau, G. and Hallot, P., 2012. Developing an ontology of space: Application to 3D city modeling. In: T. Leduc, G. Moreau and R. Billen (eds), Usage, Usability, and Utility of 3D City Models - European COST Action TU0801, EDP Sciences, Nantes, France.

Brüggemann, H. and Liebig, A., 2000. Geographic Data Infrastructure for North-Rhine Westphalia (GDI NRW). In: Proceedings of the Spatial Information Society: Shaping the Future, 6th EC-GIS Workshop, Vol. 30, Lyon, France.

Dominguez, B. D., Fernández, A. L. G. and Higueruela, F. R. F., 2011. Semantic and topological representation of building indoors: An overview. In: Proceedings of the Joint ISPRS Workshop on 3D City Modelling \& Applications and the 6th $3 D$ GeoInfo Conference, Wuhan, China.

Hagedorn, B., Trapp, M., Glander, T. and Döllner, J., 2009. Towards an Indoor Level-of-Detail Model for Route Visualization. In: 2009 Tenth International Conference on Mobile Data Management: Systems, Services and Middleware, Taipei, Taiwan, pp. 692-697.

\footnotetext{
${ }^{7}$ http://www.indoorreality.com/

${ }^{8} \mathrm{https}: / /$ www.applanix.com/products/timms-indoor-mapping.htm

${ }^{9} \mathrm{https}: / /$ matterport.com/
} 
Hsu, M.-H. and Hsu, Y.-L., 2005. Generalization of two- and three-dimensional structural topology optimization. Engineering Optimization 37(1), pp. 83-102.

Hu, Y., 2018. 1.07 - Geospatial Semantics. In: B. Huang (ed.), Comprehensive Geographic Information Systems, Elsevier, Oxford, pp. 80-94.

Jung, H.-J., Kang, H. and Lee, J., 2016. The Concepts of Level of Detail in 3D Indoor Models. In: FIG Working Week 2016 Proceedings, Christchurch, New Zealand.

Kang, H.-Y. and Lee, J., 2014. A Study on the LOD(Level of Detail) Model for Applications based on Indoor Space Data. Journal of the Korean Society of Surveying, Geodesy, Photogrammetry and Cartography 32(2), pp. 143-151.

Kang, H. Y., Hwang, J. R. and Hong, C. H., 2013. A Study on the Development of an Indoor Level of Detail(LOD) Model for the Linkage between BIM and GIS: Focusing on the Indoor Facility Management. Journal of Korea Spatial Information Society 21(5), pp. 73-82.

Kemec, S., Zlatanova, S. and Duzgun, S., 2012. A new LoD definition hierarchy for 3D city models used for natural disaster risk communication tool. In: Proceedings of the 4th International Conference on Cartography and GIS, Vol. 2, Albena, Bulgaria, pp. 17-28.

Löwner, M.-O., Benner, J., Gröger, G. and Häfele, K.-H., 2013. New Concepts for Structuring 3D City Models - An Extended Level of Detail Concept for CityGML Buildings. In: Computational Science and Its Applications - ICCSA 2013, Ho Chi Minh City, Vietnam, pp. 466-480.

Luebke, D., Reddy, M., Cohen, J. D., Varshney, A., Watson, B. and Huebner, R. (eds), 2003. Level of Detail for 3D Graphics. The Morgan Kaufmann Series in Computer Graphics, Morgan Kaufmann, San Francisco.

Monmonier, M. S., 1996. How to Lie with Maps. 2nd edn, University of Chicago Press, Chicago.

Nyerges, T. L., 1991. Analytical Map Use. Cartography and Geographic Information Systems 18(1), pp. 11-22.

Open Geospatial Consortium, 2012. OGC City Geography Markup Language (CityGML) Encoding Standard. Technical Report OGC 12-019, version 2.0.0.

Reinhardt, J. and Bedrick, J., 2016. 2016 Level of Development Specification. BIMForum.

Slocum, T. A., McMaster, R. B., Kessler, F. C. and Howard, H. H., 2010. Thematic Cartography and Geovisualization. Pearson Prentice Hall.

Solou, D. and Dimopoulou, E., 2016. Investigating the capabilities of semantic enrichment of 3D CityEngine data. In: Fourth International Conference on Remote Sensing and Geoinformation of the Environment (RSCy2016), Vol. 9688, Cyprus.

Succar, B., 2009. Building information modelling framework: A research and delivery foundation for industry stakeholders. Automation in Construction.

Tah, J., Oti, A. and Abanda, F., 2017. A state-of-the-art review of built environment information modelling (BeIM). Organization, Technology and Management in Construction: an International Journal 9(1), pp. 1638-1654.

Zhu, K. and Heun, M. H. J., 2017. Teaching and Learning of Chinese History in Minecraft: A Pilot Case-Study in Hong Kong Secondary Schools. In: Proceedings of the 2017 Conference on Interaction Design and Children, IDC '17, ACM, New York, NY, USA, pp. 405-410.
Zlatanova, S. and Isikdag, U., 2017. 3D Indoor Models and Their Applications. In: Encyclopedia of GIS, Springer, Cham, pp. 920.

Zlatanova, S., Li, K.-J., Lemmen, C. and van Oosterom, P., 2016. Indoor abstract spaces : Linking IndoorGML and LADM. In: Proceedings of the 5th international FIG workshop on 3D cadastres, Athens, Greece.

Zlatanova, S., Sithole, G., Nakagawa, M. and Zhu, Q., 2013. Problems in indoor mapping and modelling. In: The International Archives of the Photogrammetry, Remote Sensing and Spatial Information Sciences, Volume XL-4/W4, 2013. 